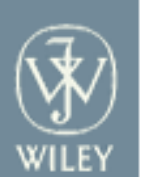

Earthquake Engineering and Structural Dynamics

\title{
Semi-Explicit Rate-Dependent Modeling of Damage Avoidance Steel Connections Using HF2V Damping Devices
}

\begin{tabular}{|r|l|}
\hline Journal: & Earthquake Engineering and Structural Dynamics \\
\hline Manuscript ID: & EQE-09-0280.R2 \\
\hline Datey - Manuscript type: & Research Article \\
\hline Author: & n/a \\
\hline & $\begin{array}{l}\text { Complete List of Authors: } \\
\text { Engineering } \\
\text { Engineering } \\
\text { Chase, J.; University of Canterbury, Mechanical Engineering }\end{array}$ \\
\hline Keywords: & $\begin{array}{l}\text { Steel Connection, HF2V devices, Non-linear viscous damping, } \\
\text { Damage-Avoidance Design, Rocking Connection, Lead damping } \\
\text { devices }\end{array}$ \\
\hline
\end{tabular}




\title{
Semi-Explicit Rate-Dependent Modeling of Damage Avoidance Steel Connections Using HF2V Damping Devices
}

\author{
Geoffrey W. Rodgers ${ }^{1}$, John B. Mander ${ }^{2}$, J. Geoffrey Chase ${ }^{3}$
}

\begin{abstract}
A rate-dependent modeling technique is developed for moment resisting steel connections that utilize non-linear viscous dampers. First, a model of the Maxwell-type is developed that considers the non-linear viscous damper and connection flexibility for translational motion. This model is compared to experimental results at several input motion frequencies to validate the results. The model is then extended to represent an exterior steel beam-to-column connection using damage avoidance design and non-linear viscous dampers. By including terms to represent structural member and connection flexibility, using appropriate geometric transformations the model can be formulated to give the overall lateral load-drift structural performance. Validation analysis shows good agreement between experimental observations and the model predictions.
\end{abstract}

\footnotetext{
${ }^{1}$ Visiting Researcher, Zachry Dept. of Civil Engineering, Texas A\&M Univ., College Station, Texas 77843-3136, USA, and PhD Student, Dept. of Mechanical Engineering, University of Canterbury, Christchurch 8140, New Zealand

2 Inaugural Zachry Professor, Department of Civil Engineering, Texas A\&M Univ., College Station, Texas 77843, USA

3 Professor, Dept. of Mechanical Engineering, University of Canterbury, Christchurch 8140, New Zealand
} 


\section{INTRODUCTION}

In recent research, high force-to-volume (HF2V) lead based dampers have been developed that can provide large resistive forces and maintain compact outer dimensions (Rodgers et al. 2007; Rodgers et al. 2008a). These devices have been implemented into several large-scale experiments, using both jointed-precast concrete and steel beam-to-column rigid connections (Mander et al. 2009; Rodgers et al. 2008b). During these experimental investigations it has become apparent that the influence of connecting elements has a large effect on the connection rigidity and energy dissipation capacity of the damping system. Specifically, any flexibility and take-up of the connecting elements reduces the displacements induced in the damper, thereby reducing the ability of the damper to efficiently dissipate energy. Therefore, to obtain the optimal behavior it is of importance to reduce the damper connection flexibility as much as possible.

Despite efforts to reduce the flexibility, the connecting elements will always influence the damper behavior. Moreover, practical limitations on shaft and connection sizes dictate that a measure of connection flexibility will always exist and have some effect on overall behavior. Therefore, it is of importance to develop a model that incorporates both the fundamental damper mechanics, while also incorporating the effects of connection flexibility.

Previous research has looked at overall flexibility components of steel connections and how they relate to subassembly deformation and fatigue considerations (Mander et al. 1994). Other research has investigated experimental testing and modeling the response of post-tensioned steel connections (Garlock et al. 2007; Pekcan et al. 2000), but has not investigated the use of non-linear viscous dampers within the connections of a steel moment frame.

There has been previous research that has investigated the response of viscoelastic damping for seismic mitigation in structural applications, and for industrial applications of vibration isolation 
(Hwang and Ku 1997; Makris and Constantinou 1991; Shen and Soong 1995). The previous research typically utilizes fractional derivatives and frequency domain analysis to model the damper properties and define the overall response. These techniques provide good agreement with experimental results, and are typically applied for damper elements that exhibit viscoelastic properties. Makris and Constantinou (1991) investigated spring-viscous damper systems, and their application for both seismic and vibration isolation. Hwang and $\mathrm{Ku}$ (1997) use system identification methods and fractional derivatives to model high-damping rubber bearings for seismic isolation of bridge decks. Hwang and $\mathrm{Ku}$ (1997) also discusses the historical lack of time-history analysis techniques for high-damping rubber bearings, due to their complex characteristics, and the traditional use of equivalent linear models. Fractional derivatives have also been used to analyse free damped vibrations of suspension bridges (Rossikhin and Shitikova 1998), and to model the effects of temperature on the energy dissipation of viscoelastic dampers (Aprile et al. 1997).

Although similar in basic concept to several of these applications, the damper system presented herein is a series system of a non-linear viscous damper with an elastic spring element. One of the main advantages of this approach, when compared to simpler models that do not incorporate the spring flexibility, is the ability to model small cyclic reversals in the loading profile. Small reversals in the loading profile cannot generally be dealt with meaningfully by models that do not include the spring flexibility and operate solely on the displacement input into the damper element, rather than the displacement input into overall damping system including associated flexibility.

The analysis presented herein utilizes time domain analysis to provide the model solution, and represents a different approach to the previous work. The previous research utilizes fractional derivatives in the analysis, where this research uses a standard first derivative raised to a fractional 
power. As such, the time domain approach was selected to easily incorporate the non-linear effects of the damper element into the analysis. The mechanics of the individual damper and spring elements of the damping system are relatively straightforward and well recognized. However, producing a rate-dependent, time-domain solution that is straight-forward to implement is not so elementary. The interdependence of the variables makes the system quite difficult to solve and the solution technique is the merit of this paper. Each component of the damper model is relatively elementary, but the model solution and robustness is unique.

This paper describes the non-linear viscous damper and flexibility model, and also explains the incorporation of this model into a broader structural sense, by incorporating the damper model into a steel beam-column moment frame connection. This overall model can be used to determine the overall connection behavior from damper, connection and member properties, using a rational mechanics based approach.

\section{TRANSIENT MODEL OF DAMPER BEHAVIOUR}

Consider a non-linear HF2V damper (Rodgers et al; 2007, 2008a) connected in series with a linear axial spring to give a system of the Maxwell type, which is schematically presented in Figure 1. The displacement across the spring is defined as $x$, the displacement across the damper is $y$, and the total displacement across the system is defined as $z$. The system is formulated such that $z$ is a known input displacement as a function of time, and the values of $x, y$, and the overall system force, $F$, are calculated.

Due to the series nature of the system the displacements and velocities (using dot notation) will sum, therefore:

$$
\begin{aligned}
& x+y=z \\
& \dot{x}+\dot{y}=\dot{z}
\end{aligned}
$$


The applied force, $F_{D}$, must also equal the spring force $(K x)$ and the damper force. Using the Pekcan et al (1999) model for non-linear viscous dampers:

$$
F_{D}=\frac{x}{f_{D}}=C_{\alpha}|\dot{y}|^{\alpha} \operatorname{sgn}(\dot{y})
$$

in which $f_{D}=$ the axial flexibility of the spring component of the damper assembly $\left(f_{D}=1 / K\right)$, where $K$ is the spring stiffness of the damper assembly; $C_{\alpha}=$ a damper constant; $\alpha=$ the velocity exponent; and $\dot{y}=$ the velocity across the damper. Substituting Equation (2) into Equation (1) and re-arranging gives:

$$
\dot{y}=\dot{z}-f_{D} \dot{F}=\left(\frac{F}{C_{\alpha}}\right)^{\left(\frac{1}{\alpha}\right)}
$$

where $\dot{F}=d F / d t$.

Herein a numerical solution approach is used to solve the coupling between Equations (1) and (3). Using backward differences, a numerical solution for Equation (3) can be obtained from:

$$
\begin{aligned}
& \dot{y}_{i+1}=\frac{z_{i+1}-z_{i}+f_{D} F_{i}}{\Delta t+f_{D} C_{\alpha}^{(1 / \alpha)}\left|F_{i+1}\right|^{1-1 / \alpha}} \\
& F_{i+1}=C_{\alpha}\left|\dot{y}_{i+1}\right|^{\alpha} \operatorname{sgn}\left(\dot{y}_{i+1}\right)
\end{aligned}
$$

The coupling of Equations (4) and (5) requires that these equations must be solved in a predictorcorrector sense. For the initial step the first predictor of force is given by:

$$
F_{1}=\frac{z_{1}}{f_{D}}
$$


And for all subsequent steps the following has been found to be a suitable predictor:

$$
F_{i+1}=F_{i}+\frac{\Delta t}{f_{D}}\left(2 \dot{x}_{i}-\dot{x}_{i-1}\right)
$$

From this solution the value of the system force on each successive time step can be obtained. This result is then used to give the value of the spring displacement, $x$, as well as the damper displacement, $y$, and their derivatives.

This predictor-corrector approach provides an accurate solution to the system equations (without iteration) as long as the time step is kept small, as it is only conditionally stable. If the time step is sufficiently small, then the predictor-corrector set of equations can be utilized, by implementing the predictor step of Equation (7), followed by the corrector step defined in Equations (4) and (5). Although this approach provides accurate results and is relatively easy to implement, accuracy can be improved if they are solved iteratively. If a larger time step is used, and iteration is necessary, then the predictor step of Equation (7) should be used, followed by repeated applications of Equations (4) and (5) until the difference between successive approximations is within a predetermined tolerance. Providing the time step used is appropriately small, convergence is rapid. The next time step can then be simulated using the same predictor and iterative corrector steps.

If larger time steps are used and iteration is necessary, an appropriate root finding numerical method may also be used to solve Equations (4) and (5). The predictor-corrector method presented above is arguably the least expensive computationally, but care must be taken not to use an unsuitable time step. The model results using the predictor-corrector model presented in Figure $2 \mathrm{~b}$ are validated against the exact solution using a MATLAB ${ }^{\mathrm{TM}}$ root finding algorithm and selection of the appropriate, real-valued, root. The exact solution from this method is presented overlaid on the 
$0.01 \mathrm{~Hz}$ results in Figure $2 \mathrm{~b}$. It is evident that both methods give the same solution, validating the use of the predictor-corrector formulation.

Figure 3 presents the response of the predictor-corrector model in Equations (4) to (7) to a $0.01 \mathrm{~Hz}$ sinusoidal displacement input. Figure $3 \mathrm{a}$ shows the response with a $0.05 \mathrm{~s}$ time step, and Figure $3 \mathrm{~b}$ shows the response with a $0.125 \mathrm{~s}$ time step. Figure $3 \mathrm{~b}$ presents the onset of instability in the numerical solution due to the increased time step. The instability is observed to be self-damping with notable oscillation converging back onto the correct solution. The specific time step required to achieve a stable solution depends on the frequency of loading and relative magnitude of system parameters, but any instability can be easily observed. The large, rapid oscillation within the unstable solution provides a clear indication of the onset of instability which will be clearly seen during analysis of simulation results.

\section{Damper Model Validation}

Experimental results of a velocity-dependent HF2V device are now used to validate the above force-displacement analytical model. The HF2V device was tested at a range of different velocities in a DARTEC ${ }^{\mathrm{TM}}$ universal testing machine to characterize the damper constant, $C_{\alpha}$, and the velocity exponent, $\alpha$. Figure 4 presents the experimental set-up for the HF2V device characterization. Two independent sets of measurements were recorded. The internal load-cell and LVDT displacement transducer were recorded as well as the measurements of a load-cell connected in series with the device and a potentiometer across the stressed part of the device shaft.

The force readings should show only minor differences, but the displacement measurements are somewhat different. The reason for this difference is that the HF2V device potentiometer measured actual device motion as well as elastic deformation of the stressed length of the shaft. The internal LVDT of the DARTEC testing machine measured the device displacement, shaft 
deformation, as well as the deformation of all the device mounts, locating bolts and the main actuator.

The experimental data was used to characterize the velocity exponent, by taking the force at peak velocity for a range of different period sine-wave input motions. Using this data, a trend-line is fitted to characterize the damper constant, $C_{\alpha}$, as well as the velocity exponent $\alpha$. The velocity exponent has been investigated in two different studies, using different HF2V devices (Mander et al. 2009; Rodgers et al. 2008a). Both studies show the device velocity exponent was typically in the range of $\alpha=0.11$ to 0.12 . This result is within the same range previously found in the characterization on much larger lead extrusion damping devices (Cousins and Porritt 1993). It should be noted that the testing speed shown in the results of Figure 2 are relatively slow due to limitations in the experimental testing equipment available. However, in the work of Cousins and Porritt (1993), tests on the larger dampers were undertaken up to velocities of approximately $1 \mathrm{~m} / \mathrm{s}$ with closely similar results for the velocity component, with $\alpha=0.12$.

Figure 2 presents results of experimental tests at two different input sine-wave frequencies of 0.01 and $0.0025 \mathrm{~Hz}$ along with the analytical modeling of the HF2V device response. When applying the model in Equations (1) to (7), the following parameters were used, $\alpha=0.12, C_{\alpha}=170$, $f_{D}=3.3 \times 10^{-3} \mathrm{~mm} / \mathrm{kN}$. From Figure 2 it is evident that overall good agreement exists between the experimental behavior and the analytical model prediction.

\section{ROTATIONAL FORMULATION FOR MOMENT FRAME CONNECTIONS}

\section{Structural Flexibility}

Recent research has investigated the use of HF2V devices in a steel beam-to-column moment frame connection of the variety presented in Figure 5 (Mander et al. 2009). The top-hung steel flange (beam) connection can be modeled as a rotational version of the linear (translational) damper model 
presented above. The presence of the beam and column in effect provide additional sources of flexibility. Thus, in extending the behavioral model to capture the rotational effects, the structural elements must first be considered, without the displacement/rotation contributions from the damper displacement, $y$. If the damper is locked, such that $y=0$, then the flexibility contributions of the structural elements can be determined.

The total displacement at the top of the column shown in Figure 5a becomes a combination of displacement contributions from the column, the beam, and the connection rotation due to damper connection flexibility and angle flexibility. This relationship can be written as:

$$
\Delta_{T}=\Delta_{c}+\Delta_{b}+\Delta_{x}
$$

where $\Delta_{T}=$ the total displacement due to all flexibility effects; $\Delta_{c}=$ the displacement contribution from column flexibility; $\Delta_{b}=$ the contribution from beam flexibility; and $\Delta_{x}=$ the contribution due to the connection rotation as a result of damper connection and top angle flexibility, and shear deformation of the column panel zone.

Each of these individual contributions can be individually defined based on well-known structural mechanics. The drift angle due to column deflection is:

$$
\theta_{C}=\frac{V_{C} L_{C}^{2}}{12 E I_{C}}
$$

Where $V_{c}=$ the column base-shear; $L_{c}=$ defined as the column height, as shown in Figure 5a; and $E I_{c}=$ the column rigidity

Similarly, the beam rotation, $\theta_{b}$, due to the beam shear is given as:

$$
\theta_{b}=\frac{V_{b} L_{b}^{2}}{3 E I_{b}}
$$


where $V_{b}=$ the beam shear; $L_{b}=$ the clear beam length, as shown in Figure 5a; and $E I_{b}=$ the beam rigidity.

From moment equilibrium of the subassembly, the beam shear can be defined:

$$
V_{b}=V_{C} \frac{L_{C}}{L}
$$

where $L=$ the distance from the column centerline to the beam strut location, as shown in Figure 5a.

This relationship can then be used to define the beam rotation in terms column shear:

$$
\theta_{b}=V_{C} \frac{L_{b}^{2}}{3 E I_{b}} \frac{L_{c}}{L}
$$

During elastic deformation the beam rotation will be equal to the column rotation, so no further transformation is required.

The rotation of the column due to the overall joint flexibility components, $f_{D}$, can be determined based on simple geometric transformations. For a given damper force, $F_{D}$, the displacement across the connecting elements, $x$, is given by Equation (2) $\left(x=f_{D} F_{D}\right)$. From this displacement, $x$, the column rotation can be defined as:

$$
\theta_{x}=\frac{f_{D} F_{D}}{j D}
$$

where $f_{D}=$ the flexibility associated with the damper connections, shear deformation of the panel zone, and deflection of the top angle; $j D=$ the internal lever arm between the top angle/flange-hung connection and the line of the re-action of the damper, as shown schematically in Figure 5a. Moment equilibrium of the beam requires:

$$
F_{D}=\frac{V_{b} L_{b}}{j D}
$$


This result can be combined with Equation (11) to give column shear as a function of the damper resistance:

$$
V_{C}=F_{D} \frac{j D}{L_{C}} \frac{L}{L_{b}}
$$

Substituting Equation (15) into Equation (13) gives the column rotation as a function of column base-shear:

$$
\theta_{x}=V_{C} \frac{f_{D} L_{b} L_{c}}{(j D)^{2} L}
$$

All of these components can now be summed to obtain the overall elastic structural drift, $\theta_{S}$, as a function of column base-shear:

$$
\theta_{S}=V_{c} f_{\theta}
$$

where $f_{\theta}=$ the total rotational elastic flexibility, defined as:

$$
f_{\theta}=f_{\theta c}+f_{\theta b}+f_{\theta x}
$$

where the results of Equations (9), (12), and (16) are divided by the column base-shear to obtain the flexibility and substituted into Equation (18) to give:

$$
f_{\theta}=\frac{L_{C}^{2}}{12 E I_{c}}+\frac{L_{C} L_{b}^{2}}{3 L E I_{b}}+\frac{f_{D} L_{b} L_{C}}{(j D)^{2} L}
$$

\section{Connection Flexibility}

In Equation (19) the connection flexibility, $f_{D}$, encompasses components from the damper and its connection elements. This can be expanded as follows: 


$$
f_{D}=f_{t r}+f_{d r}+f_{a a}+f_{a f}+f_{s}
$$

in which $f_{t r}=$ the axial flexibility associated with the damper tie-rods/connecting rods and load-cell; $f_{d r}=$ the axial flexibility associated with the retaining bolts across the damper, which stretch during damper action; $f_{a a}=$ the axial flexibility of the bottom leg of the angle connection; $f_{a f}=$ the flexibility associated with flexure/bending of the angle bracket during loading; and $f_{s}=$ the flexibility associated with the column shear across the column panel zone. These components of flexibility are defined as follows.

The tie-rod flexibility is:

$$
f_{t r}=\frac{L_{t r}}{E A_{t r}}
$$

where $L_{t r}=$ the stressed length of the damper shaft and axial elements connecting the damper to the column face; and $A_{t r}=$ the total cross-sectional area of the tie-rods/damper shaft.

The damper rods/retaining bolts that hold the damper end-caps together elongate during tensile loading of the damper, with flexibility:

$$
f_{d r}=\frac{L_{d r}}{E A_{d r}}
$$

where $L_{d r}=$ the stressed length of the damper retaining bolts; and $A_{d r}=$ the total cross-sectional area of the damper-rods/retaining bolts.

The axial flexibility of the bottom leg of the angle connection is:

$$
f_{a a}=\frac{L_{a a}}{E A_{a a}}
$$


where $L_{a a}=$ the effective stressed length of the bottom leg of the angle connection; and $A_{a a}=$ the cross-sectional area of the bottom leg of the angle.

The flexibility associated with the flexural deformation/bending of the top angle is:

$$
f_{a f}=\frac{L_{a f}^{3}}{C E I_{a f}}
$$

in which $L_{a f}=$ the effective length of the top leg of the angle that is undergoing bending; $I_{a f}=$ the second moment of area of the top leg of the angle about the axis of bending; and $C=$ a constant dependent on the assumption used for the boundary conditions, where $C=3$ for fixed-free boundary conditions and $C=12$ for fixed-fixed boundary conditions.

The flexibility from shear deformation is given as:

$$
f_{S}=\frac{j D}{G t_{w} D_{c o l}}
$$

where $j D=$ as previously defined, the distance between the damper line of action and the top flange connection; $G=$ the shear modulus of the column material; $t_{w}=$ the thickness of the column web; and $D_{c o l}=$ the total depth of the column.

Damper motion, $y$, results in small angle rigid body rotation component that must also be considered in addition to the elastic components. The rotation of the column due to damper motion can be determined from:

$$
\theta_{y}=\frac{y}{j D}
$$

where $\theta_{y}=$ the drift angle due to damper displacement $y$, and in derivative form as: 


$$
\dot{\theta}_{y}=\frac{\dot{y}}{j D}
$$

Thus from Equations (2) and (15), the column base-shear can now be defined as a function of damper properties:

$$
V_{C}=F_{D}\left(\frac{j D L}{L_{b} L_{C}}\right)=\left(\frac{j D L}{L_{b} L_{C}}\right) C_{\alpha}|\dot{y}|^{\alpha} \operatorname{sgn}(\dot{y})
$$

Or in terms of rotational velocity using Equation (27):

$$
V_{c}=C_{\theta}\left|\dot{\theta}_{y}\right|^{\alpha} \operatorname{sgn}\left(\dot{\theta}_{y}\right)
$$

where:

$$
C_{\theta}=\frac{j D L}{L_{b} L_{C}} C_{\alpha}(j D)^{\alpha}
$$

\section{Numerical Implementation}

The model terms for the moment-rotation structural system are analogous to the force-deformation (translational) damper system model that was previously presented and validated above. The flexibility terms, as expressed in Equations (17) and (19) are analogous to the spring flexibility, $f_{D}$, in the simple translational model. Likewise, the rotation component from damper motion, $\dot{\theta}_{y}$, defined in Equation (27) is analogous to the damper term, $y$, in the damper model. Therefore, the numerical predictor-corrector solution can be modified to utilize these common facets, and thus be re-formulated to define a relationship between joint rotation and column base-shear. From Equation (7) it follows that the predictor step is: 


$$
V_{c, i+1}=V_{c, i}+\frac{\Delta t}{f_{\theta}}\left(2 \dot{\theta}_{S, i}-\dot{\theta}_{S, i-1}\right)
$$

During the initial few time-steps, a different approximation must be used, as the backward difference values may not be available for application. Therefore, during these initial few steps, the base-shear can be obtained by considering that initial deformation will be essentially governed by the elastic regime only, thus:

$$
V_{C, i}=\frac{\theta_{z, i}}{f_{\theta}}
$$

where $\theta_{z}=$ the total known input rotation to the system; and $f_{\theta}=$ the total elastic flexibility as defined previously in Equation (19).

The corrector step takes a very similar form to that previously presented for the simplified model presented in Equations (4) and (5), and is defined:

$$
\dot{\theta}_{y, i}=\frac{\theta_{z, i+1}-\theta_{z, i}+\theta_{S, i}}{\Delta t+f_{\theta} C_{\theta}^{1 / \alpha}\left|V_{C, i}\right|^{1-1 / \alpha}}
$$

This new value of $\dot{\theta}_{y, i}$ can then be used as a corrector step, to re-calculate the system force, $V_{c, i}$, using:

$$
V_{c, i}=C_{\theta}\left|\dot{\theta}_{y, i}\right|^{\alpha} \operatorname{sgn}\left(\dot{\theta}_{y, i}\right)
$$

As before, this system of equations can be iterated to converge onto a solution within each timestep. Alternatively, this predictor-corrector approach can be used without iteration, with the limitation that it is only conditionally stable and must utilize a sufficiently fine time-step. 


\section{JOINT OPENING-CLOSING: MODELING STIFFNESS SWITCHING}

When a top flange-hung beam-to-column moment connection (of the type shown in Figure 5) opens and closes, different stiffnesses are observed (Mander et al. 2009). When the connection is undergoing a loading portion of the response, and the sign of the velocity is the same sign as the force, the vertical leg of the top angle is being subjected to single bending, thus a value of $C=3$ is applicable in Equation (24). Conversely, when the connection is being unloaded and the sign of the velocity is different to the sign of the force, the vertical leg of the top angle is being subjected to double bending, thus a value of $C=12$ is applicable in Equation (24). Based on the above arguments and supported by experimental observations, under one completely reversed cycle of loading the stiffness/flexibility of the connection will switch when these criteria are met. A switching function has been devised to model this phenomenon:

$$
f_{\theta}=\left(\frac{f_{\theta 1}+f_{\theta 2}}{2}\right)+A\left(\frac{f_{\theta 1}-f_{\theta 2}}{2}\right) \tanh \left[50\left|\theta_{Z, i}-\theta_{\text {reset }}\right|-\pi\right]
$$

where $f_{\theta 1}=$ the overall flexibility when $C=3$; and $f_{\theta 2}=$ the overall flexibility when $C=12$. In Equation (35) a constant of 50 was found to provide a suitable rate of change of the stiffness, and the $\operatorname{argument} A$ is calculated at each time step, and is defined:

$$
A=\operatorname{sgn}\left(\left(\theta_{Z, i}-\theta_{Z, i-1}\right)\left(V_{C, i-1}\right)\right)
$$

where the argument $A$ can be monitored for a change, and when the change is detected, the current value of $\theta_{Z}$ is defined as the reset drift, $\theta_{\text {reset }}$. The reset displacement is then used to define the change in flexibility in Equation (35). 


\section{BEAM-COLUMN JOINT MODEL VALIDATION}

As previously described, a steel beam-column connection with top-hung angle connection was used as the experimental specimen, as shown in Figure 5. The 2D specimen utilized a cut back beam end to allow positive and negative (gap-opening and closing) joint rotations. Basic specimen dimensions, as defined in Figure 5, were $L_{c}=2 \mathrm{~m}, L=1.5 \mathrm{~m}$, using member sections 360UB44.7 and $310 \mathrm{UC} 15$ for the beam and column respectively. The HF2V device utilized was the same as that previously characterized, and shown in Figures 2 and 4. More specific specimen dimensions, different configurations, design considerations, and hysteresis results are presented in Mander et al. (2009).

The connection between the beam and column was fitted with four potentiometers to measure connection rotation and any torsion present. A photograph of the experimental setup is presented in Figure 5b; this shows the location of the three potentiometers on the south side of the setup. The top potentiometer measured angle displacement/pull-off from the column face, while the remaining potentiometers at the approximate beam mid-height, and at the damper line of action measured the connection rotation.

Ideally, the top potentiometer would be measuring at the top angle-beam flange interface. However, physical constraints restricted the position of the potentiometer stylus to be $25 \mathrm{~mm}$ below this interface. If the beam end acts with only rigid rotation and shear deformation, the potentiometer reading can be expected to be linear with eccentricity from the centre of rotation. By checking the potentiometers for this linearity, the translation of the angle bracket at the angle/flange interface can be calculated, and can be used to validate the assumptions used to predict the angle flexibility contributions. 
Figure 6a presents the experimentally observed behavior under reversed cyclic loading to drift amplitudes of $0.25,0.5,1,2,3$, and $4 \%$. These experimental results are presented only for model validation purposes and only the most important final results are presented. Mander et al. (2009) is an experimentally focused manuscript and presents more specific details, different experimental results, and connection design considerations. Figure $6 \mathrm{~b}$ shows the modeled results using Equations (31) to (34). Although good agreement between the observed and modeled results is evident, several points should be noted.

\section{DISCUSSION: THE INFLUENCE OF FLEXIBILITY ON PREFORMANCE}

\section{Damper Flexibility}

It is evident that the analytical model and experimental results show good agreement. To thoroughly validate the analytical model, each flexibility component can be individually examined based on local potentiometer data. By delineating these effects the model assumptions can be individually validated, providing a thorough validation of the analytical model, and greater insight into key design aspects of such a connection. This result enables the experimentally validated assumptions and simple rational mechanics to be used for any such connection to accurately predict the overall response.

The displacements recorded by the two potentiometers located at the damper line of action are closely similar, and indicate that no significant out-of-plane motion or torsion is present. The rigid body connection rotation can be determined from the joint potentiometers, which can determine the input value $z$ into the damper-spring system, and the elastic deflections which can experimentally determine an overall elastic subassembly stiffness.

The damper force is plotted against the connection displacement at the damper location to present the overall damper/connection system response in Figure 7a. Also plotted in Figure 7a is the 
force against the actual damper displacement, $y$, measured by a potentiometer on the unstressed portion of the damper shaft. The difference in these two plots is the result of connecting element flexibility. It is evident in Figure 7a that the connecting elements have added notable additional flexibility to the system. Figure $7 \mathrm{~b}$ shows the displacement values of $y$ and $z$ plotted against time, which correspond to the displacements in Figure 7a, and are projected downwards from Figure 7a to Figure $7 \mathrm{~b}$, using the same horizontal axes.

\section{Connection Flexibility}

The difference between the plots in Figure $7 \mathrm{~b}$ represents the flexibility components and is the effective spring displacement, $x$, as defined in Equation (1a) and plotted in Figure 8a. This result can be used to derive an experimental value of the spring flexibility $f_{D}$. The measured value of $x$ is compared to a calculated value of $x$ based on the measured damper force $F_{D}$ and Equation (2) $\left(x=f_{D} F_{D}\right)$. The value of $f_{D}$ is modified to minimize the least-squares error between the measured and calculated values of $x$. The resulting plot of the measured and calculated values of spring/connection displacement, $x$, is presented in Figure 8a. It should be noted that although the displacements presented in Figure 8a are relatively small (on the order of 1-2mm), they represent only the elastic component of the damper displacement, and must be considered in combination with the rigid-body components of displacement. Even with the relatively small displacement magnitudes, the elastic component has a major effect on the initial elastic stiffness of the damper system and therefore the initial stiffness of the subassembly. Therefore, the small outright magnitude of the elastic displacement component does not make it insignificant to the overall results.

It is evident that a single (uniform) value of $f_{D}$ may not accurately represent the experimental data. The most obvious cause is the different reaction mechanism in the top-hung 
angle connection. The angle will be in axial tension in the bottom leg and bending in the top leg during gap closing, but will act with axial compression and a form of direct bearing onto the column face during gap opening. These mechanisms have different associated flexibilities which must be reflected in the analytical model and is achieved by the previously defined addition of heuristics. Another important aspect evident in Figure 8a is that the measured and calculated values of spring displacement, $x$, follow a very similar trend for small amplitude motions. It is only for large amplitude motions that significant deviations are seen, due to the prying effects where the top angle lifts off the column face, and low level yielding of the connection elements. These additional non-linear effects are not included into the model and are the reason why the results vary for the larger amplitude motions.

The analytical value of the connection flexibility parameter, $f_{D}$, can be calculated based on the experimental dimensions. Table 1 presents the individual contributions and the overall flexibility values for the experimental set-up, as used in the model presented in Figure 6b. From these values of flexibility, the overall structural flexibility parameter, $f_{\theta}$ can be determined using Equation (19). Since the rigid body connection angle can be obtained from the joint potentiometers, the rigid body component of column displacement can be subtracted to get the elastic component. This elastic component is a function of elastic member deformations and elastic deformation of the experimental setup, such as the axial compression/tension in the beam support strut, and connection to the strong floor.

From the elastic displacement component, a total experimental elastic member flexibility can be calculated. The displacement is defined as $d=f_{\theta} V_{c o l}$ and the value of $f_{\theta}$ is adjusted to minimize the least-squares error to the calculated value, the result of which is presented in 
Figure $8 \mathrm{~b}$. As before, a single uniform stiffness value does not provide good agreement with the experimental data due to the different deformation regimes during different loading directions.

It is evident in Figure 6 that the variable stiffness is important to capture the different reaction mechanisms that are present in the experimental results. Overall, the model and experimental results show good agreement. However, the experimental results show a slight bilinear behavior that is attributed to additional friction/binding of the shaft due to the lateral loading. This lateral load is due to joint motion, which is an arc about the rocking edge, whereas the shaft can only move in a linear path. Therefore, some bending will be induced within the shaft, resulting in a variable friction force which contributes to the column base-shear. The use of clevis attachments in future tests will maintain only axial force in the damper, eliminate the bi-linear component, and show better agreement between the model and experimental results.

It is important to note that the overall connection design presented here does not lead to any inherent self-centering characteristics. The experimental results of Figure 6 show that an external force is required to return the connection to the original zero-displacement position. While other concrete and steel connections that utilize post-tensioned prestressing bars have been tested which exhibit self-centering characteristics (Garlock et al. 2007; Rodgers et al. 2008b), that is not the focus of this specimen. Therefore, as this connection design does not provide static re-centering capability, this type of connection must be considered as part of a larger structural system. If some selected connections within a structure provide a restoring, self-centering capability, then the overall structure may maintain self-centering characteristics. While these are important considerations, this type of analysis is the focus of ongoing research and is not presented here.

Another key aspect evident in Figure 6 is the large influence that the angle reaction regime and associated flexibility has on the overall elastic stiffness of the subassembly. It is advantageous 
to have a high initial stiffness and low yield drift, for a given yield force. Therefore, to stiffen the connection the angle connection is a primary consideration, and in future connections the top-hung angle may be modified to incorporate a split-tee connection (WT-shape). The angle used in the experiment was equivalent to a L6 $\times 6 \times 1 / 2$ and could be easily replaced by a WT shaped connection, such as the WT6x39.5. The flange could then be bolted to the column face above and below the top beam flange, and the web would be used for connection to the beam flange. Using such a split-tee connection would reduce the flexibility to approximately $10 \%$ of that defined for the top-hung angle under fixed-pinned conditions. This would reduce the overall flexibility by approximately $70 \%$ for that case, and by at least $25 \%$ for the fixed-fixed angle assumption. This connection detailing should result in a higher initial elastic stiffness (reduced flexibility), lower yield drift, and a more rigid structure. It is advantageous to have this higher initial stiffness to reduce structural drifts.

\section{CONCLUSIONS}

Based on the investigation described herein, the following conclusions are drawn:

1. A simple damper model was developed that accounts for connection flexibility and velocitydependence of the damper. The damper model was calibrated to experimental data, and shows good agreement with the overall behavior, as well as the velocity-dependence.

2. The damper model was then extended to a base-shear to drift relationship, by accounting for structural member flexibility and geometric transformations. The model, based entirely on rational mechanics, provides a good analytical representation of lateral load-rotation behavior, without empirically determined constants.

3. It is important to recognise the slight asymmetry that exists in connection behavior due to different reaction mechanisms of the top angle connection. To accommodate this phenomenon 
a continuous numerical switch function was developed. Implementation shows good agreement with the experimental results.

4. Assessment of the flexibility components for all structural elements provides insight into both model prediction and design improvements for future connections. Individual consideration of the member flexibility effects indicates that the top angle may contribute a large amount to the total flexibility.

\section{ACKNOWLEDGEMENTS:}

The modeling work presented herein was developed while the first author was resident of Texas A\&M University on a Fulbright-EQC Graduate Award. This support, and the support of the New Zealand Tertiary Education Commission through the Bright Futures scheme, is gratefully acknowledged.

\section{REFERENCES}

Aprile, A., Inaudi, J. A., and Kelly, J. M. (1997). "Evolutionary Model of Viscoelastic Dampers for Structural Applications." Journal of Engineering Mechanics, 123(6), pp 551-560. Doi: 10.1061/(ASCE)07339399(1997)123:6(551)

Cousins, W. J., and Porritt, T. E. (1993). "Improvements to lead-extrusion damper technology." Bulletin of the New Zealand National Society for Earthquake Engineering, 26(3), pp 342-348.

Garlock, M. M., Sause, R., and Ricles, J. M. (2007). "Behavior and design of posttensioned steel frame systems." Journal of Structural Engineering, 133(3), pp 389-399. Doi: 10.1061/(ASCE)07339445(2007)133:3(389)

Hwang, J. S., and Ku, S. W. (1997). "Analytical Modeling of High Damping Rubber Bearings." Journal of Structural Engineering, 123(8), pp 1029-1036. Doi: 10.1061/(ASCE)0733-9445(1997)123:8(1029)

Makris, N., and Constantinou, M. C. (1991). "Fractional-Derivative Maxwell Model for Viscous Dampers." Journal of Structural Engineering, 117(9), pp 2708-2724. Doi: 10.1061/(ASCE)07339445(1991)117:9(2708)

Mander, J. B., Chen, S. S., and Pekcan, G. (1994). "Low-cycle fatigue behavior of semi-rigid top-and-seat angle connections." Engineering Journal, 31(3), pp 111-122.

Mander, T. J., Rodgers, G. W., Chase, J. G., Mander, J. B., MacRae, G. A., and Dhakal, R. P. (2009). "A Damage Avoidance Design Steel Beam-Column Moment Connection Using High-Force-To-Volume Dissipators." ASCE Journal of Structural Engineering, 135(11), pp 1390-1397. Doi: 10.1061/(ASCE)ST.1943-541X.0000065 
Pekcan, G., Mander, J. B., and Chen, S. S. (1999). "Fundamental considerations for the design of non-linear viscous dampers." Earthquake Engineering and Structural Dynamics, 28(11), pp 1405-1425. Doi: 10.1002/(SICI)1096-9845(199911)28:11<1405::AID-EQE875>3.0.CO;2-A

Pekcan, G., Mander, J. B., and Chen, S. S. (2000). "Experiments on steel MRF building with supplemental tendon system." Journal of Structural Engineering, 126(4), pp 437-444. Doi: 10.1061/(ASCE)07339445(2000)126:4(437)

Rodgers, G. W., Chase, J. G., Mander, J. B., Leach, N. C., and Denmead, C. S. (2007). "Experimental development, tradeoff analysis and design implementation of high force-to-volume damping technology." Bulletin of the New Zealand Society for Earthquake Engineering, 40(2), pp 35-48.

Rodgers, G. W., Mander, J. B., Chase, J. G., Dhakal, R. P., Leach, N. C., and Denmead, C. S. (2008a). "Spectral analysis and design approach for high force-to-volume extrusion damper-based structural energy dissipation." Earthquake Engineering \& Structural Dynamics, 37(2), pp 207-223. Doi: 10.1002/eqe.752

Rodgers, G. W., Solberg, K. M., Mander, J. B., Chase, J. G., Bradley, B. A., Dhakal, R. P., and Li, L. (2008b). "Performance Of A Damage-Protected Beam-Column Subassembly Utilizing External HF2V Energy Dissipation Devices." Earthquake Engineering \& Structural Dynamics, 37(13), pp 1549-1564. Doi: DOI: 10.1002/eqe.830

Rossikhin, Y. A., and Shitikova, M. V. (1998). "Application of Fractional Calculus for Analysis of Nonlinear Damped Vibrations of Suspension Bridges." Journal of Engineering Mechanics, 124(9), pp 10291036. Doi: 10.1061/(ASCE)0733-9399(1998)124:9(1029)

Shen, K. L., and Soong, T. T. (1995). "Modeling of Viscoelastic Dampers for Structural Applications." Journal of Engineering Mechanics, 121(6), pp 694-701. Doi: 10.1061/(ASCE)0733-9399(1995)121:6(694) 
Table 1: Damper/Connection Flexibility Components

\begin{tabular}{|l|l|l|}
\hline \multicolumn{1}{|c|}{ Flexibility Component } & $\begin{array}{c}\text { Calculated Value } \\
(\mathbf{x ~ 1 0} \mathbf{~} \mathbf{m m} / \mathbf{k N})\end{array}$ & Equation \\
\hline Angle-flexure: $f_{a f}=\frac{L_{a f}{ }^{3}}{C E I_{a f}}$ & $15.7(3.9)^{*}$ & $(24)$ \\
\hline Angle-axial: $f_{a a}=\frac{L_{a a}}{E A_{a a}}$ & 0.1 & $(23)$ \\
\hline Shear: $f_{S}=\frac{j D}{G t_{w} D_{c o l}}$ & 1.0 & $(25)$ \\
\hline Damper-rods: $f_{d r}=\frac{L_{d r}}{E A_{d r}}$ & 0.5 & $(22)$ \\
\hline Tie-rod: $f_{t r}=\frac{L_{t r}}{E A_{t r}}$ & 3.2 & $(20)$ \\
\hline Total Flexibility: $f_{D}$ & $20.4(8.6)^{*}$ & \\
\hline$*$ for C = 3 and $(12)$. & & \\
\hline
\end{tabular}




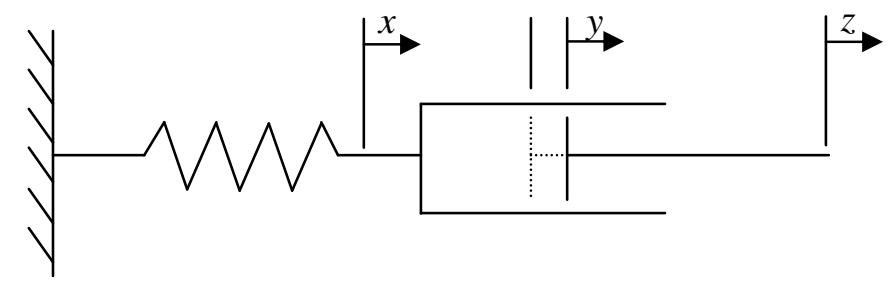

Figure 1: Schematic diagram of the damper-spring system 


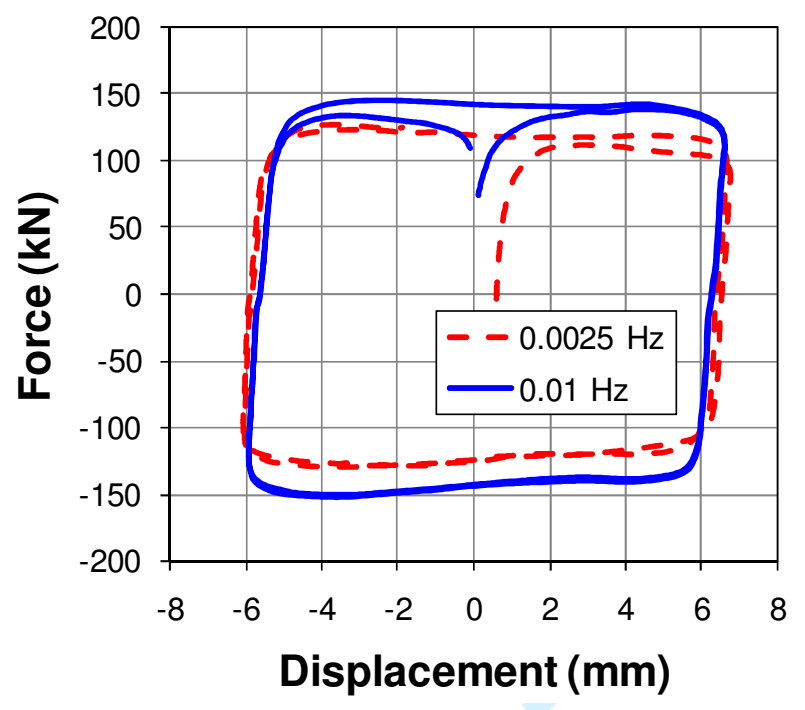

a) Experimental Results

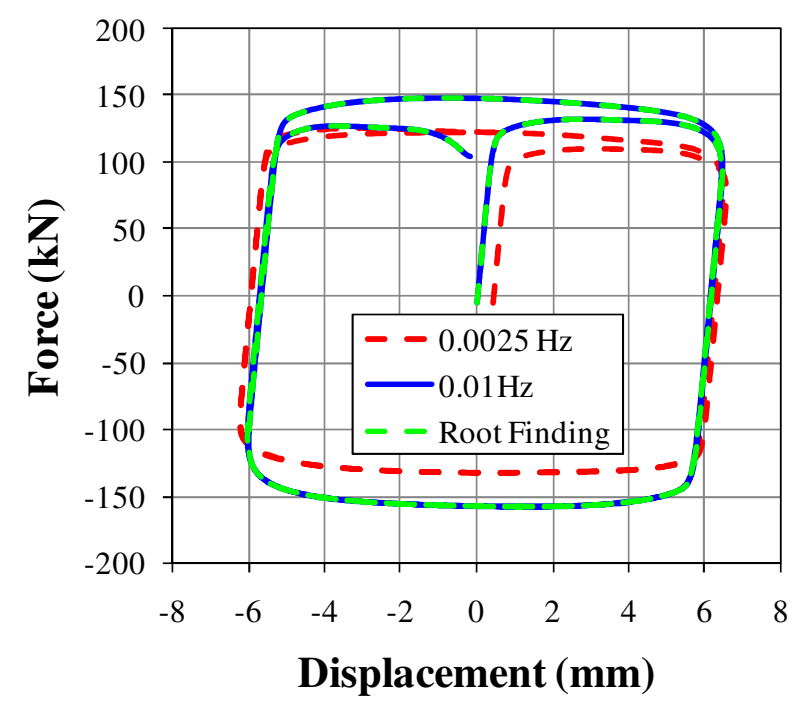

b) Model Results

Figure 2: Comparison of Modeled and Experimental results 


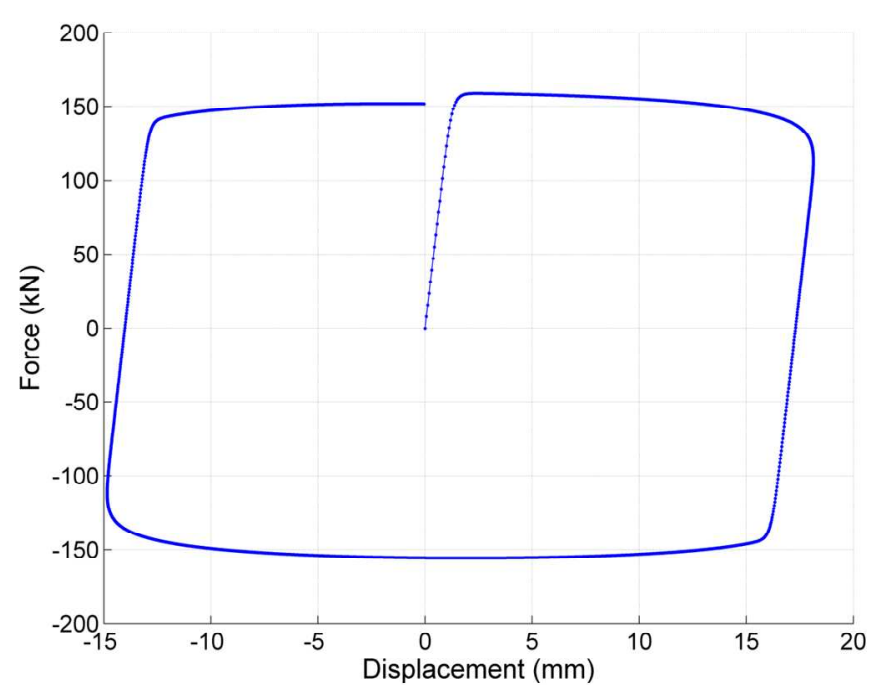

a) Stable model solution with $\Delta t=0.02 \mathrm{~s}$

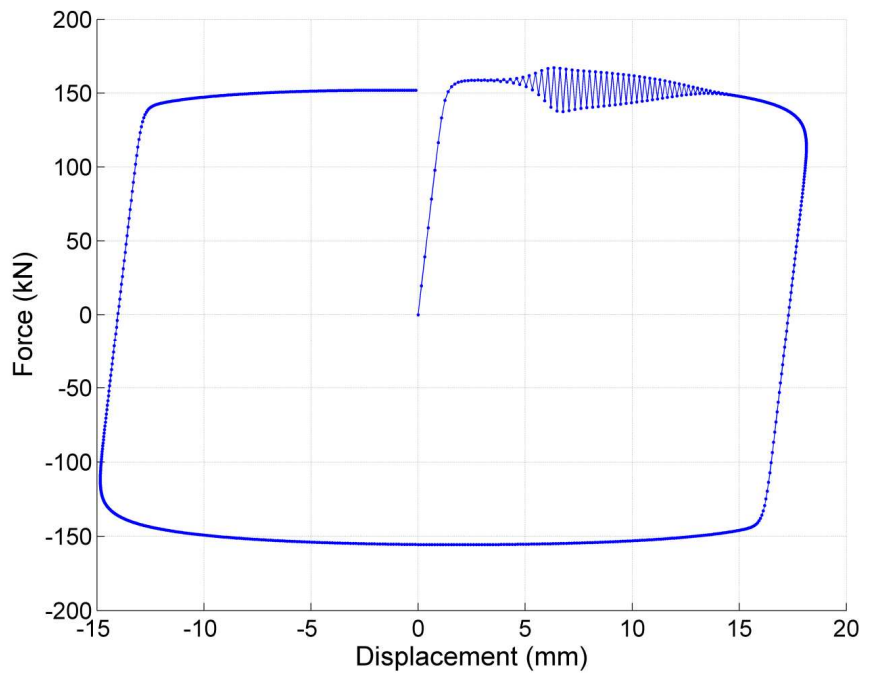

b) Unstable model solution with $\Delta t=0.125 \mathrm{~s}$

Figure 3: Onset of instability for the damper model. 


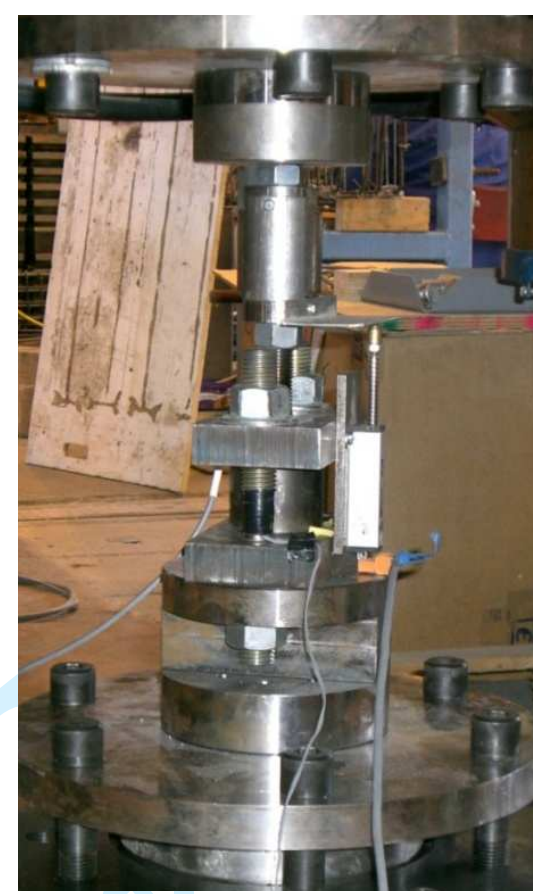

Figure 4: Experimental set-up for HF2V device characterization 


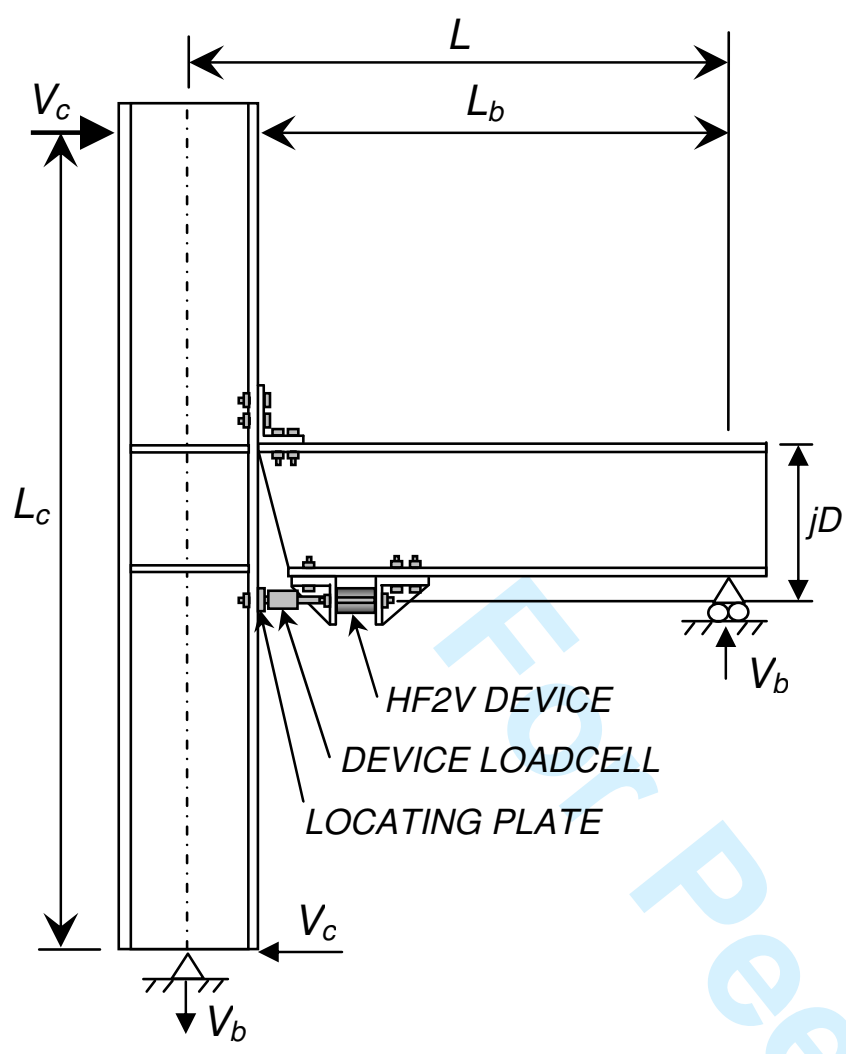

a) Experimental subassemblage

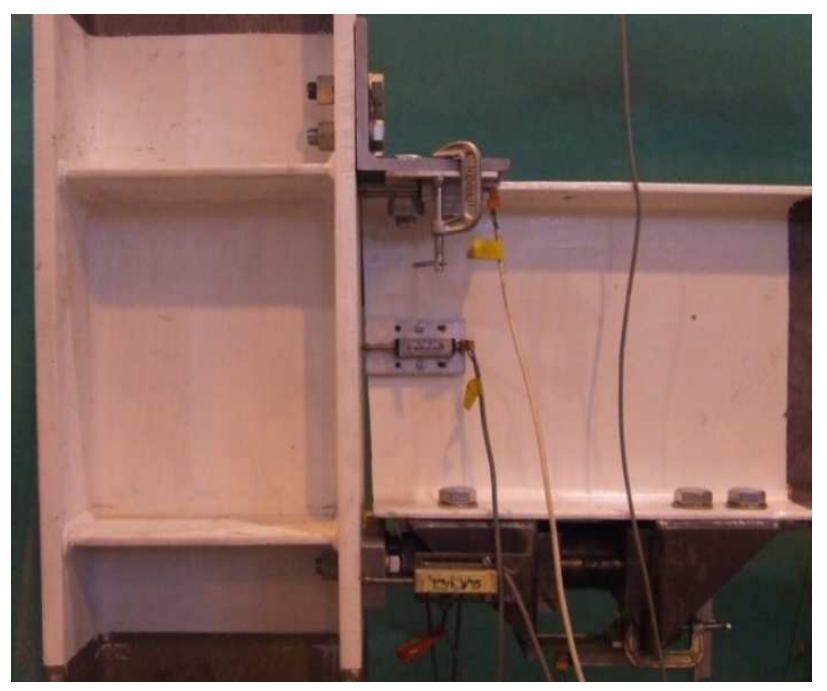

b) Photograph of joint with damper

Figure 5: Steel beam-to-column connection with HF2V device 


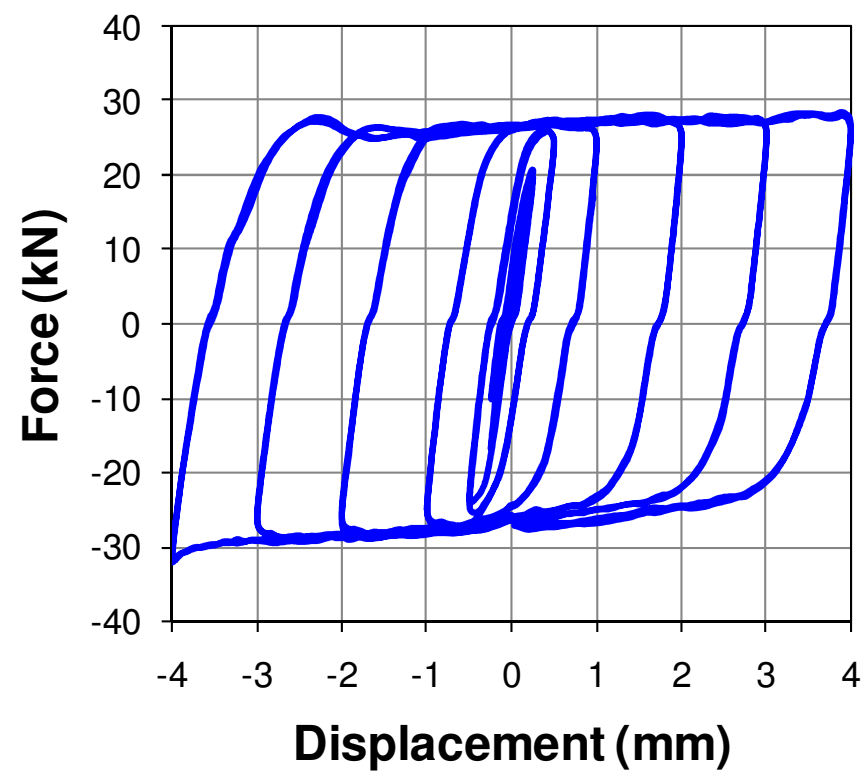

a) Experimental Results

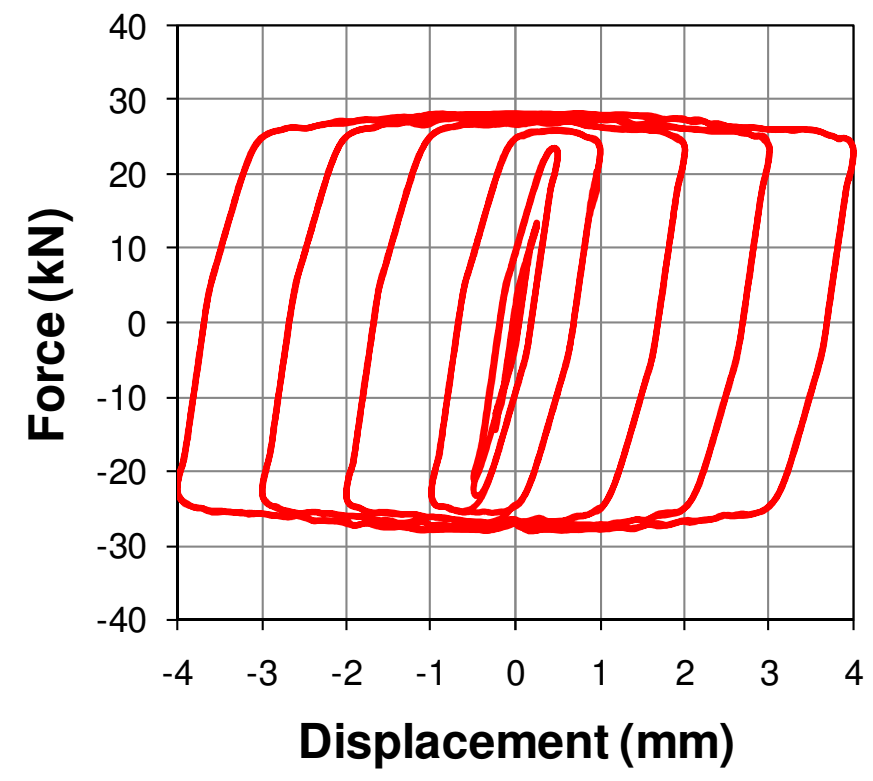

b) Model Results

Figure 6: Comparative results for the overall subassembly performance 
a)

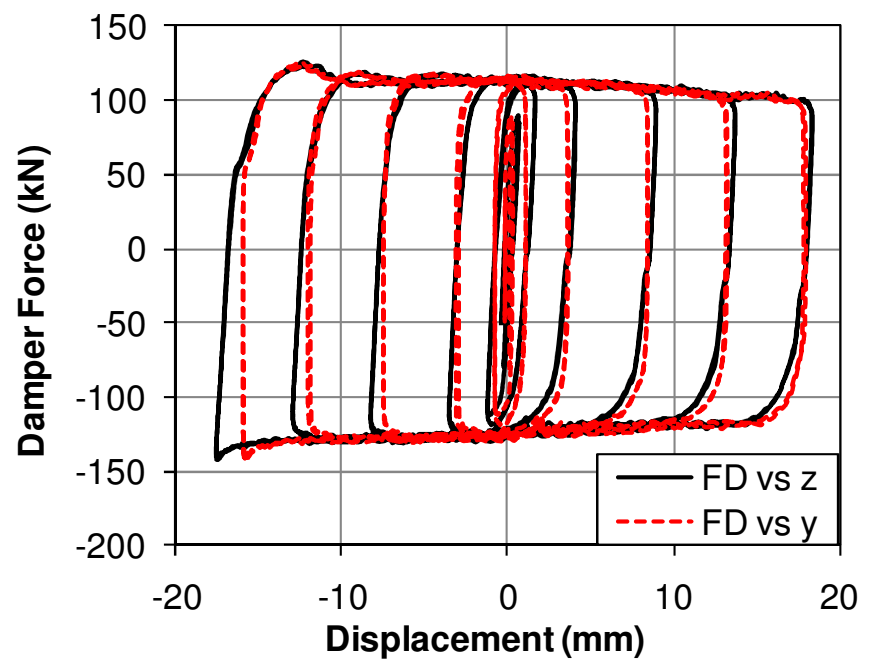

b)

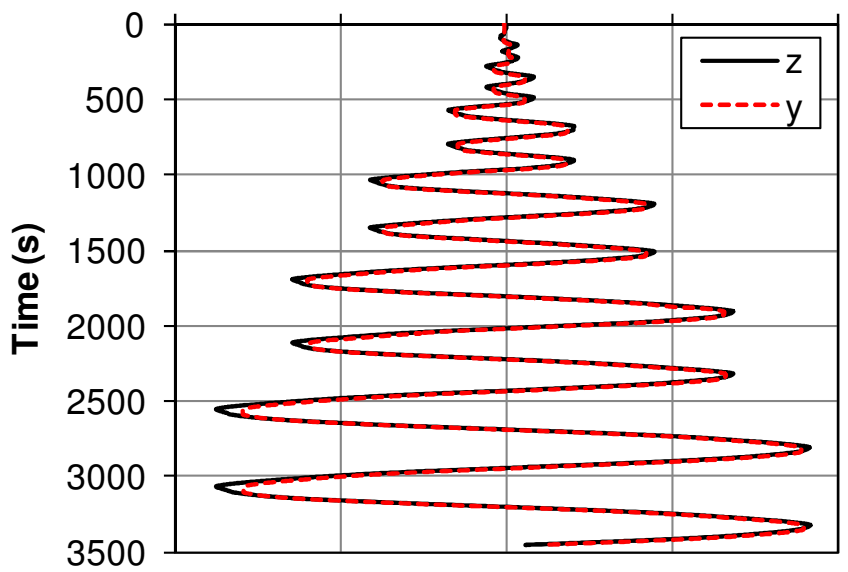

Figure 7: a) The damper force $F_{D}$ plotted against the damper displacement $y$, and joint displacement at damper location, $z$, and b) the damper and joint displacements corresponding to those in a). 


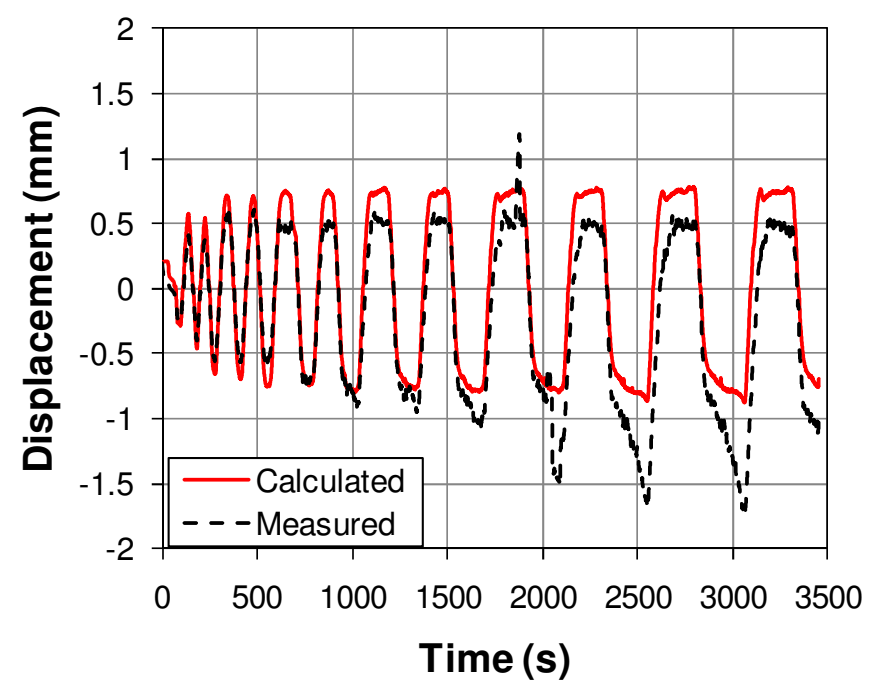

a) spring/connection displacement, $x(=z-y)$

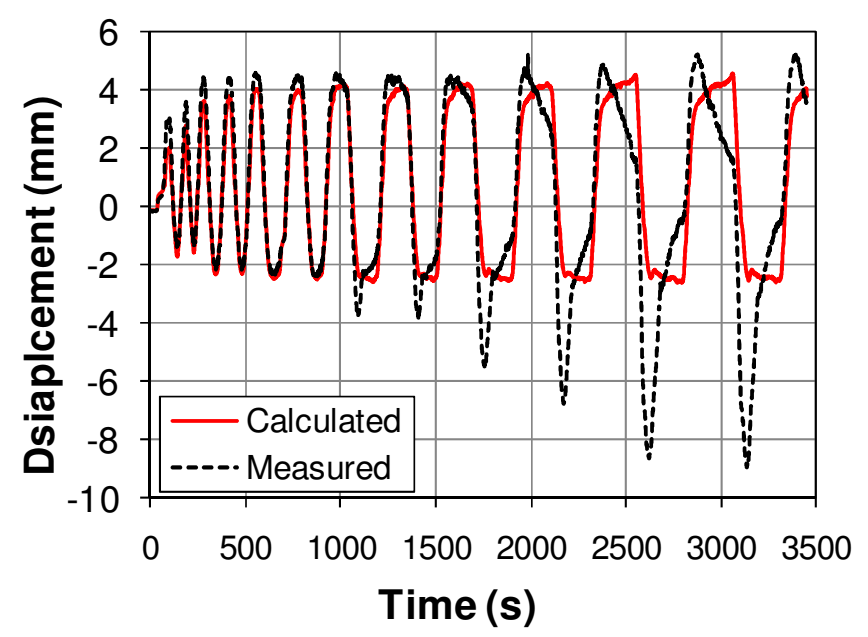

b) elastic structural displacement component

Figure 8: Experimentally measured and calculated elastic displacement values 